\title{
CRYO-BALLOON RECONSTRUCTION FROM TWO VIEWS
}

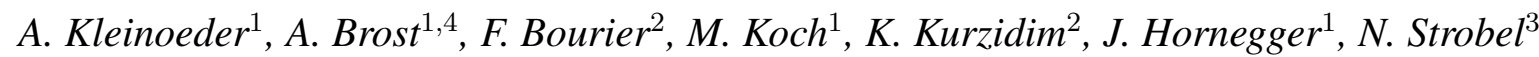 \\ ${ }^{1}$ Pattern Recognition Lab, University of Erlangen, Erlangen, Germany \\ ${ }^{2}$ Klinik für Herzrhythmusstörungen, Krankenhaus Barmherzige Brüder, Regensburg, Germany \\ ${ }^{3}$ Siemens AG, Healthcare Sector, Forchheim, Germany \\ ${ }^{4}$ Alexander.Brost@informatik.uni-erlangen.de
}

\begin{abstract}
Atrial fibrillation is a major cause of stroke. Its treatment is performed under fluoroscopic image guidance. Augmented fluoroscopy has become a useful tool during the ablation procedure for navigation under X-ray. Unfortunately, current navigation systems do not provide tools to localize and visualize a cryo-balloon catheters in 3-D. This is why we present a new approach to reconstruct the cryo-ballon catheter, modeled as a sphere, from two views. The reconstruction result can then be overlayed onto live fluoroscopic images during the procedure. In simulation studies, we compared our technique to a reference method. While both methods worked equally well on noise-free data, we found our method more reliable if the input data was affected by noise. For example, in the presence of noise with a standard deviation of $4 \mathrm{~mm}$, our maximum 3-D reconstruction error was less than $1 \mathrm{~mm}$.
\end{abstract}

Index Terms - Atrial Fibrillation, Augmented Fluoroscopy, Reconstruction

\section{INTRODUCTION}

Atrial fibrillation (AFib), the most common sustained arrhythmia, is a major cause of stroke $[1,2]$. One treatment option for AFib is the electrical isolation of the four pulmonary veins attached to the left atrium by catheter ablation [3]. It is recommended, e.g., when patients fail drug therapy. C-arm $\mathrm{X}$-ray systems are used for AFib procedures. Augmented fluoroscopy using a perspectively forward projected overlay representation of 3-D objects onto live fluoroscopic images has become a useful tool procedure for navigation when performing ablation procedures $[4,5,6]$. Using a modern biplane C-arm system with two imaging planes, not only visualization but also 3-D localization and object reconstruction has become possible. Two risk factors of the current radio-frequency catheter ablation approach are pulmonary vein stenosis and esophageal fistula. To reduce these risks, cryo-balloon catheter ablation techniques can be used [7, 8]. Unfortunately, current navigation tools do not provide tools to localize and visualize cryo-balloon catheters in 3-D. In our approach, the catheter is modeled as a sphere in 3-D. Methods to reconstruct ellipsoids either require three views [9] or additional 3-D information [10]. A method for sphere reconstruction has already been proposed in [11], but it turns out to be rather sensitive to noise. This is why we present a novel method to reconstruct a cryo-balloon catheter from two views. It is well suited to compute a 3-D model even in the presence of noise. Used as part of a fluoroscopic overlay image for augmented fluoroscopy applications, we expect that our method can further increase the safety and effectiveness of the cryo-balloon ablation approach.

\section{SPHERE RECONSTRUCTION}

The reconstruction process consists of two steps [11]. In the first steps, the 2-D input data is processed and in the second step, the 3-D catheter model is generated. As input, manually selected 2-D points on the boundary of the balloon-catheter in both views are needed, see Figure 1 for an example of a single view. These points are denoted as $\mathbf{p}_{A, i}, \mathbf{p}_{B, j} \in \mathbb{R}^{2}$ with $i, j \in \mathbb{N}$ for imaging plane $A$ and imaging plane $B$. The points hold image coordinates of plane $A \mathbf{p}_{A, i}=(u, v)^{T}$ and plane $B \mathbf{p}_{B, j}=(u, v)^{T}$ with $u, v, \in \mathbb{R}$. Two-dimensional ellipses are fitted to these points according to [12], resulting in the implicit ellipse parameters $a_{A}, b_{A}, c_{A}, d_{A}, e_{A}, f_{A} \in \mathbb{R}$ for imaging plane $A$ and $a_{B}, b_{B}, c_{B}, d_{B}, e_{B}, f_{B} \in \mathbb{R}$ for imaging plane $B$. The implicit equations can then be written as

$$
\begin{array}{r}
a_{A} u^{2}+b_{A} u v+c_{A} v^{2}+d_{A} u+e_{A} v+f_{A}=0 \\
a_{B} u^{2}+b_{B} u v+c_{B} v^{2}+d_{B} u+e_{B} v+f_{B}=0
\end{array}
$$

with the 2-D image coordinates $u, v \in \mathbb{R}$. These equations can be reformulated in matrix notation as

$$
\begin{aligned}
\tilde{\mathbf{p}}_{A, i}^{T} \mathbf{C}_{A} \tilde{\mathbf{p}}_{A, i} & =0 \\
\tilde{\mathbf{p}}_{B, j}^{T} \mathbf{C}_{B} \tilde{\mathbf{p}}_{B, j} & =0
\end{aligned}
$$


with $\tilde{\mathbf{p}}_{A, i}=\left(\mathbf{p}_{A, i}, 1\right)^{T}$ and $\tilde{\mathbf{p}}_{B, j}=\left(\mathbf{p}_{B, j}, 1\right)^{T}$ and the matrices

$$
\begin{aligned}
& \mathbf{C}_{A}=\left(\begin{array}{ccc}
a_{A} & \frac{b_{A}}{2} & \frac{d_{A}}{2} \\
\frac{b_{A}}{2} & c & \frac{e_{A}}{2} \\
\frac{d_{A}}{2} & \frac{e_{A}}{2} & f_{A}
\end{array}\right) \\
& \mathbf{C}_{B}=\left(\begin{array}{ccc}
a_{B} & \frac{b_{B}}{2} & \frac{d_{B}}{2} \\
\frac{b_{B}}{2} & c & \frac{e_{B}}{2} \\
\frac{d_{B}}{2} & \frac{e_{B}}{2} & f_{B}
\end{array}\right) .
\end{aligned}
$$

Using the implicit ellipse parameters, 3-D cones $\mathbf{Q}_{A}, \mathbf{Q}_{B} \in$ $\mathbb{R}^{4 \times 4}$ can be calculated by incorporating the projection matrices $\mathbf{P}_{A}, \mathbf{P}_{B} \in \mathbb{R}^{3 \times 4}[13,14]$

$$
\begin{aligned}
\mathbf{Q}_{A} & =\mathbf{P}_{A}^{T} \mathbf{C}_{A} \mathbf{P}_{A} \\
\mathbf{Q}_{B} & =\mathbf{P}_{B}^{T} \mathbf{C}_{B} \mathbf{P}_{B} .
\end{aligned}
$$

Now, the ray from the optical center through the center of the fitted ellipse is calculated. In the following, we present the calculation only for plane $A$. The 3-D cones have a structure as follows

$$
\mathbf{Q}_{A}=\left(\begin{array}{cc}
\tilde{\mathbf{Q}}_{A} & \mathbf{a}_{A} \\
\mathbf{a}_{A}^{T} & \mu_{A}
\end{array}\right)
$$

with the left upper diagonal sub-matrix $\tilde{\mathbf{Q}}_{A} \in \mathbb{R}^{3 \times 3}$, the vector $\mathbf{a}_{A} \in \mathbb{R}^{3}$ and the scalar value $\mu_{A} \in \mathbb{R}$. If a cone has its tip at the origin of the coordinate system and its main axis aligns with one of the coordinate axes, we get [11]

$$
\begin{aligned}
& \mathbf{a}_{A}=0 \\
& \mu_{A}=0 .
\end{aligned}
$$

To align an arbitrary cone with the coordinate system, we have to find a transformation

$$
\mathbf{T}_{A}=\left(\begin{array}{cc}
\mathbf{R}_{A} & \mathbf{t}_{A} \\
0 & 1
\end{array}\right)
$$

with the rotation $\mathbf{R}_{A} \in \mathbb{R}^{3 \times 3}$ and the translation $\mathbf{t}_{A} \in \mathbb{R}^{3}$. The transformed cone $\hat{\mathbf{Q}}_{A} \in \mathbb{R}^{4 \times 4}$ is then given by [11]

$$
\begin{gathered}
\hat{\mathbf{Q}}_{A}=\mathbf{T}_{A}^{T} \mathbf{Q}_{A} \mathbf{T}_{A} \\
=\left(\begin{array}{cc}
\mathbf{R}_{A}^{T} \tilde{\mathbf{Q}}_{A} \mathbf{R}_{A} & \mathbf{R}_{A}^{T}\left(\tilde{\mathbf{Q}}_{A} \mathbf{t}_{A}+\mathbf{a}_{A}\right) \\
\left(\mathbf{t}_{A}^{T} \tilde{\mathbf{Q}}_{A}+\mathbf{a}_{A}^{T}\right) \mathbf{R}_{A} & \mathbf{t}_{A}^{T} \tilde{\mathbf{Q}}_{A} \mathbf{t}_{A}+2 \mathbf{a}_{A}^{T} \mathbf{t}_{A}+b
\end{array}\right) .
\end{gathered}
$$

Considering the first element of $\hat{\mathbf{Q}}_{A}$, the rotation matrix $\mathbf{R}_{A}$ is given by the matrix of eigenvectors of $\tilde{\mathbf{Q}}_{A}$. Using Equation (10), the translation is given as

$$
\mathbf{t}_{A}=-\tilde{\mathbf{Q}}_{A}^{-1} \mathbf{a}_{A} .
$$

Given the rotation and translation, the central rays $\mathbf{r}_{A}, \mathbf{r}_{B} \in$ $\mathbb{R}^{3}$ for both cones can be calculated and intersected by the method presented in [15] to find the center of the reconstructed sphere $\mathbf{w}_{c} \in \mathbb{R}^{3}$. To calculate the radius of the reconstructed sphere, each cone is intersected with a 3-D

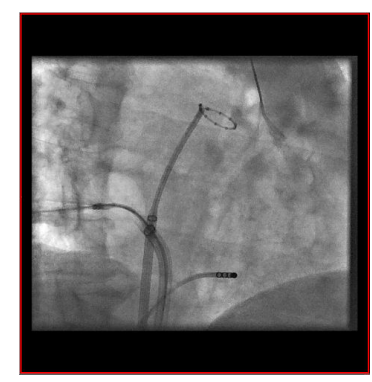

(a)

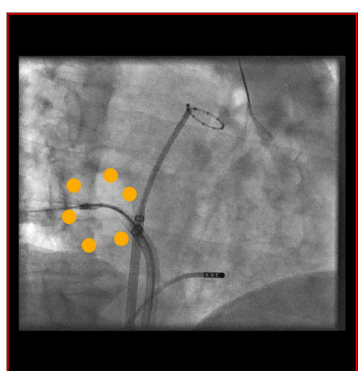

(b)
Fig. 1. (a) The original fluoroscopic image during a regular atrial fibrillation procedure using a cryo-balloon catheter. (b) The manually selected points of the balloon-catheter are superimposed onto the live fluoroscopic image (orange).

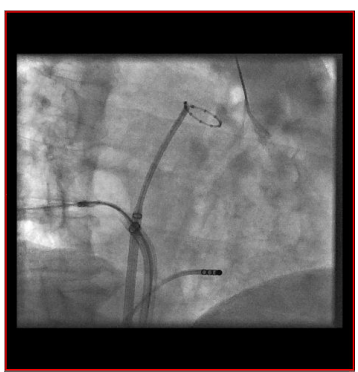

(a)

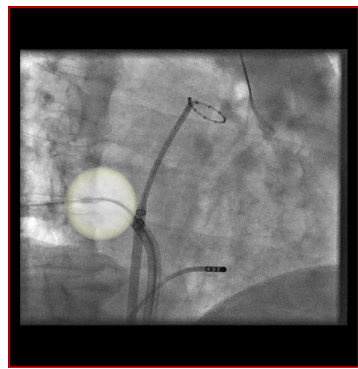

(b)
Fig. 2. (a) The original fluoroscopic image during a regular atrial fibrillation procedure using a cryo-balloon catheter at the lower right pulmonary vein. (b) The same image with the reconstructed balloon-catheter as 3-D overlay.

plane parallel to the imaging plane at the position of the calculated center $\mathbf{w}_{c}$. This method is part of the 3-D ellipse reconstruction method presented in $[13,14]$. The intersection of a 3-D cone with a 3-D plane yields an ellipse in 3-D, especially in our case. The 3-D points of the ellipse lie on the sphere. Each point is now used to calculate the radius as described in [11]. The mean of all calculated radii yields the spheres' radius resulting from one cone. Averaging the radius results for both cones results the spheres' final radius.

\section{EVALUATION AND RESULTS}

For evaluation of our method, we performed a simulation study and performed experiments using a real biplane Carm X-ray system. For the simulation, we computed 250 spheres each at a different positions in 3-D space within a maximum distance to the center of the volume of $150 \mathrm{~mm}$ and with a radius between $5 \mathrm{~mm}$ and $15 \mathrm{~mm}$. The simulation was performed using ideal $\mathrm{C}$-arm projection matrices as described in $[15,16]$. The $\mathrm{C}$-arm positions were chosen to be $90^{\circ}$ apart which is similar to a clinical setup. We 


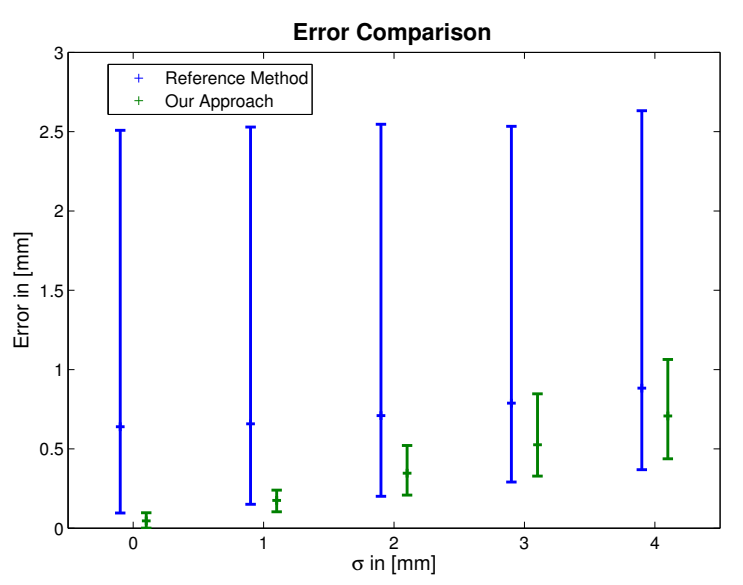

Fig. 3. Reconstruction results of our approach in comparison to the reference method [11]. The proposed method is more robust in the presence of noise (see green graphics).

compared our approach to the reference method in [11] in the presence of Gaussian noise with a standard deviation $\sigma \in[0 \mathrm{~mm}, 1 \mathrm{~mm}, 2 \mathrm{~mm}, 3 \mathrm{~mm}, 4 \mathrm{~mm}]$. The results are shown in Figure 3.

The experiments were performed on an Artis zee biplane C-arm X-ray system (Siemens AG, Healthcare Sector, Forchheim, Germany) at a clinical partner site (Krankenhaus Barmherzige Brüder, Regensburg, Germany). We used a cryo-ballon catheter placed in a bucket filled with contrast agent to evaluate our 3-D sphere reconstruction from two views. At first, a C-arm computed tomography data set (syngo DynaCT, Siemens AG, Forchheim, Germany) was acquired to obtain a 3-D data set [17] of the object. Afterwards, biplane fluoroscopic images were taken, the 2-D input points for our algorithm were manually selected, and sphere reconstruction from two views was performed. The reconstruction result was visually compared to the 3-D data set, see Figure 4. The associated 2-D images are shown in Figure 4.

\section{DISCUSSION AND CONCLUSIONS}

During the course of this work, we found our sphere reconstruction method from two views robust and easy to use. We contribute the better performance of our method to two facts. First, we do not use principal component analysis (PCA) for ellipse fitting. PCA is of advantage if many samples are present that facilitate a robust parameter estimate. In our case, we only require a few points set along object boundaries. Usually five to seven points are sufficient. In such a case, the PCA approach turns out to be very sensitive especially if the samples are not equally distributed along the object boundary. Second, we do not rely on only one or two frontier points, as proposed in [11]. Instead, we use about 100 points to determine the radius of the sphere in 3-D. Thanks to

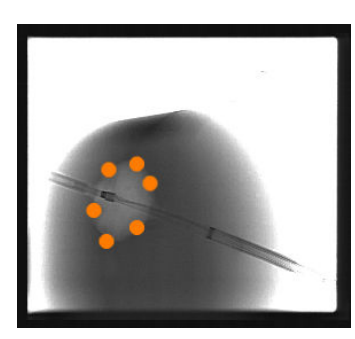

(a) Plane A

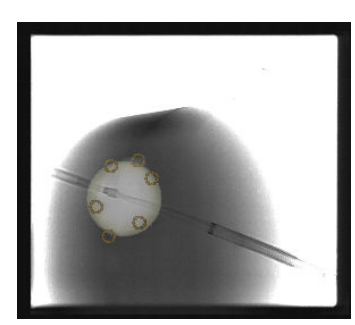

(c) Plane A

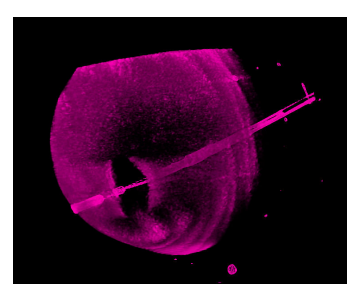

(e) 3-D Data Set

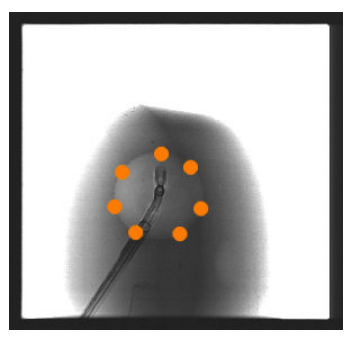

(b) Plane B

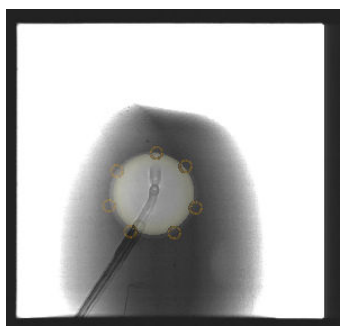

(d) Plane B

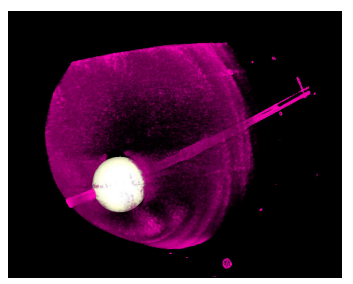

(f) Reconstruction in 3-D
Fig. 4. (a) Fluoroscopic image in plane $A$ with manually selected 2-D points on the boundary of the cryo-balloon catheter as input for the reconstruction method. (b) The same as in (a) for imaging plane $B$. (c) The reconstructed sphere model is superimposed on the fluoroscopic image shown in (a). (d) The same is shown for plane $B$. (e) C-arm computed tomography of a small bucket filled with contrast agent in which the inflated cryo-balloon catheter was placed. (f) The reconstruction of the catheter is shown together with the volumetric data set. For visualization, the volume data is colored in purple.

these improvements, our 3-D reconstruction is more robust to noise.

The proposed method is likely to be helpful during cryoballoon catheter ablation procedures as it provides visual feedback to the physician, e.g., about previous balloon positions when multiple freezing treatments are applied to the same pulmonary vein. In the next steps, we are going to clinically evaluate the proposed approach to get a better understanding of its utility for cryo-balloon catheter treatments. We expect that the use of our approach will further improve the safety and efficacy of this treatment option. 


\section{REFERENCES}

[1] P. Wolf, R. Abbott, and W. Kannel, "Atrial fibrillation as an independent risk factor for stroke: the Framingham Study," Stroke, vol. 22, pp. 983-988, 1991.

[2] C. Fuller and M. Reisman, "Stroke Prevention in Atrial Fibrillation: Atrial Appendage Closure," Curr Cardiol Rep, vol. [Epub ahead of print], pp. n/a, 2011.

[3] H. Calkins, J. Brugada, D. Packer, et al., "HRS/EHRA/ECAS Expert Consensus Statement on Catheter and Surgical Ablation of Atrial Fibrillation: Recommendations for Personnel, Policy, Procedures and Follow-Up," Heart Rhythm, vol. 4, no. 6, pp. 1-46, Jun. 2007.

[4] S. De Buck, F. Maes, J. Ector, J. Bogaert, S. Dymarkowski, H. Heidbüchel, and P. Suetens, "An augmented reality system for patient-specific guidance of cardiac catheter ablation procedures," IEEE Trans. Med. Imaging, vol. 24, no. 11, pp. 1512-1524, 2005.

[5] J. Ector, S. De Buck, W. Huybrechts, D. Nuyens, S. Dymarkowski, J. Bogaert, F. Maes, and H. Heidbüchel, "Biplane three-dimensional augmented fluoroscopy as single navigation tool for ablation of atrial fibrillation: Accuracy and clinical value," Heart Rhythm, vol. 5, no. 7, pp. 957-964, Mar. 2008.

[6] J. Ector, S. De Buck, W. Coudyzer F. Maes D. Loeckx, S. Dymarkowski, J. Bogaert, and H. Heidbüchel, "Changes in Left Atrial Anatomy Due to Respiration: Impact on Three-Dimensional Image Integration During Atrial Fibrillation Ablation," J Cardiovasc. Electr., vol. 19, no. 7, pp. 828-834, 2008.

[7] B. Avitall, D. Urboniene, G. Rozmus, D. Lafontaine, R. Helms, and A. Urbonas, "New Cryotechnology for Electrical Isolation of the Pulmonary Veins," Journal of Cardiovascular Electrophysiology, vol. 14, no. 3, pp. 281-286, 2003.

[8] Y. Van Belle, P. Janse, M. Rivero-Ayerza, A. Thornton, E. Jessurun, D. Theuns, and L. Jordaens, "Pulmonary vein isolation using an occluding cryoballoon for circumferential ablation: feasibility, complications, and short-term outcome," European Heart Journal für Kardiologie, vol. 28, pp. 2231 - 2237, 2007.

[9] S. Ma and L. Li, "Ellipsoid Reconstruction from Three Perspective Views," Pattern Recognition, International Conference on, vol. 1, pp. 344, 1996.

[10] S. Wijewickrema, A. Paplinski, and C. Esson, "Reconstruction of ellipsoids on rollers from stereo images using occluding contours," in International Conference on Computer Vision Theory and Applications, Setubal, Portugal, February 2006.

[11] S. Wijewickrema, A. Paplinski, and C. Esson, "Reconstruction of Spheres using Occluding Contours from Stereo Images," in Proceedings of the 18th International Conference on Pattern Recognition (ICPR'06), 2006.

[12] R. Halir and J. Flusser, "Numerically Stable Direct Least Squares Fitting Of Ellipses," in WSCG, 1998, pp. 253-257.

[13] A. Brost, R. Liao, J. Hornegger, and N. Strobel, “3D Respiratory Motion Compensation during EP Procedures by Image-Based 3-D Lasso Catheter Model Generation and Tracking," in Lect. Notes Comput. Sci., G.Z. Yang, D. J. Hawkes, D. Rueckert, J. A. Noble, and C. J. Taylor, Eds., London, 2009, vol. 5761, pp. 394401.

[14] A. Brost, R. Liao, N. Strobel, and J. Hornegger, "Respiratory motion compensation by model-based catheter tracking during EP procedures," Medical Image Analysis, vol. 14, no. 5, pp. 695-706, 2010, Special Issue on the 12th International Conference on Medical Image Computing and Computer-Assisted Intervention (MICCAI) 2009 .

[15] A. Brost, N. Strobel, L. Yatziv, W. Gilson, B. Meyer, J. Hornegger, J. Lewin, and F. Wacker, "Accuracy of Xray image-based $3 \mathrm{D}$ localization from two $\mathrm{C}$-arm views: a comparison between an ideal system and a real device," in Medical Imaging 2009: Visualization, ImageGuided Procedures, and Modeling, M. I. Miga and K. H. Wong, Eds., Lake Buena Vista, FL, USA, February 2009, vol. 7261, p. 72611Z, SPIE.

[16] A. Brost, N. Strobel, L. Yatziv, W. Gilson, B. Meyer, J. Hornegger, J. Lewin, and F. Wacker, "Geometric Accuracy of 3-D X-Ray Image-Based Localization from Two C-Arm Views," in Workshop on Geometric Accuracy In Image Guided Interventions - Medical Image Computing and Computer Assisted Interventions 2009, L. Joskowicz, P. Abolmaesumi, and M. Fitzpatrick, Eds., London UK, September 2009, pp. 12-19, MICCAI.

[17] N. Strobel, O. Meissner, J. Boese, T. Brunner, B. Heigl, M. Hoheisel, G. Lauritsch, M. Nagel, M. Pfister, E.P. Rührnschopf, B. Scholz, B. Schreiber, M. Spahn, M. Zellerhoff, and K. Klingenbeck-Regn, "Imaging with Flat-Detector C-Arm Systems," in Multislice CT (Medical Radiology/Diagnostic Imaging), M. F. Reiser, C. R. Becker, K. Nikolaou, and G. Glazer, Eds., chapter 3, pp. 33-51. Springer Berlin / Heidelberg, third edition, January 2009. 